\title{
Mortalidade por câncer de mama masculino nas regiões brasileiras e nos estados do Nordeste
}

\section{Mortality due to male breast cancer in brazilian regions and in Northeastern states}

\author{
José Felipe Costa da Silva, Edson Mendes Marques, Denise Rodrigues da Silva, \\ Alisson Douglas da Nóbrega Correia, Raiana Fernanda da Silva Santos, \\ Wine Suélhi dos Santos
}

Como citar este artigo: SILVA, JOSÉ F. C.; MARQUES, EDSON M.; SILVA, DENISE R.; CORREIA, ALISSON D. N.: SANTOS, RAIANA F. S.; SANTOS, UINE S. Mortalidade por câncer de mama masculino nas regiões brasileiras e nos estados do Nordeste. Revista Saúde (Sta. Maria). 2020; 46 (2).

\section{Autor correspondente:}

Nome: José Felipe Costa da Silva

E-mail: felipedoshalom@yahoo.

com.br

Telefone: (84) 99606-7|40

Formação Profissional:

Fisioterapeuta, Mestrando em

Gestão e Inovação em Saúde pela Universidade Federal do Rio Grande do Norte (UFRN), Natal, RN, Brasil.

Filiação Institucional: Universidade Federal do Rio Grande do Norte Endereço para correspondencia: Rua: Vereador José Félix Dantas Bairro: Rainha do Prado

Cidade: Florânia

Estado: Rio Grande do Norte CEP: 59335-000

Data de Submissão:

05/08/2019

Data de aceite:

18/05/2020

Conflito de Interesse: Não há conflito de interesse

\section{(cc) $\mathrm{BY}-\mathrm{NC}-\mathrm{ND}$}

\section{RESUMO}

No Brasil, o câncer é a segunda causa de morte ficando atrás apenas das doenças cardiovasculares. 0 principal câncer encontrado nas mulheres é o de mama, entretanto em casos raros esse pode acometer pessoas do sexo masculino, levando ao óbito de forma mais rápida. Objetivo: identificar a taxa de mortalidade por câncer de mama masculino no Brasil, com ênfase nos estados da região Nordeste. Métodos: Trata-se de um estudo ecológico e temporal com abordagem quantitativa. Foram selecionados as 5 regiões brasileiras e os 9 estados da região Nordeste nos anos de 2001 a 2015. Os dados foram coletados através do site do atlas online de mortalidade do Instituto Nacional do Câncer. Resultados: 1839 homens foram a óbito em razão do câncer de mama no período citado, a partir dos 30 a 39 anos houve um aumento nas notificações, em relação ao Nordeste os estados do Ceará, Rio Grande do Norte e Sergipe apresentam as três maiores taxas. Conclusão: evidenciou-se que o Sudeste foi a região que apresentou maior número de óbitos e maior taxa. No Nordeste os estados do Ceará, Rio Grande do Norte e Sergipe permaneceram acima da média e o Maranhão teve a menor taxa de mortalidade.

PALAVRAS-CHAVE: Neoplasias da Mama Masculina; Mortalidade; Brasil.

\section{ABSTRACT}

In Brazil, cancer is the second leading cause of death behind only cardiovascular diseases. The main cancer found in women is breast cancer, however in rare cases it can affect male people, leading to death more quickly. Objective: to identify the male breast cancer mortality rate in Brazil, with emphasis on the states of the Northeast region. Methods: This is an ecological and temporal study with a quantitative approach. The 5 Brazilian regions and the 9 states of the Northeast region were selected between 2001 and 2015. Data were collected through the online mortality atlas website of the National Cancer Institute. Results: 1839 men died due to breast cancer in the period mentioned, from 30 to 39 years old there was an increase in notifications, in relation to the Northeast the states of Ceará, Rio Grande do Norte and Sergipe have the three highest rates. Conclusion: it was shown that the Southeast was the region that had the highest number of deaths and the highest rate. In the Northeast, the states of Ceará, Rio Grande do Norte and Sergipe remained above average and Maranhão had the lowest mortality rate.

KEYWORDS: Male Breast Neoplasms; Mortality; Brazil. 


\section{INTRODUÇÃO}

O câncer de mama masculino é uma neoplasia maligna rara que representa menos de $1 \%$ dos tipos de câncer em homens. Este valor também corresponde ao quantitativo de câncer de mama no mundo. Contudo, a incidência dessa morbidade está aumentando devido ao envelhecimento da população podendo alcançar 15\% em alguns grupos².

Segundo o Instituto nacional de câncer (INCA) no Brasil existem mais de 309 mil homens e 316 mil mulheres diagnosticados com câncer apenas em 2020. No sexo feminino o câncer de mama abarca 29,7\% dos diagnósticos ${ }^{3}$. Já entre os homens o câncer de mama se apresenta numa taxa menor, enquanto órgãos como pulmão, próstata, intestino, estômago e fígado são mais atingidos ${ }^{4,5}$.

O aparecimento das neoplasias está, de forma geral, associado a fatores como envelhecimento populacional, alimentação inadequada, fatores genéticos, ambientais, ocupacionais, tabagismo e sedentarismo $0^{6,7}$. Um estudo realizado por Yousef ${ }^{2}$ considera o avançar da idade, desequilíbrios hormonais, genética e exposição à radiação fatores de alto risco ao aparecimento do câncer de mama masculina.

Um ponto importante a ser considerado é que o sexo masculino apresenta maior morbidade e mortalidade e, portanto, menor expectativa de vida quando comparado ao sexo feminino, levando esses indivíduos a um estado de fragilidade. 0 envelhecimento dessa população aumenta em paralelo ao crescente surgimento de Doenças Crônicas não Transmissíveis (DCNT) e esse panorama é agravado devido a mínima procura aos serviços de saúde, ocasionando assim maiores gastos em formas de tratamento mais agressivos posteriormente ${ }^{8}$.

Outro aspecto significativo é que a população masculina busca de forma mais tardia os atendimentos de saúde. Segundo dados da pesquisa nacional por amostra de domicílios (PNAD) 20,8\% dos homens e 10,1\% das mulheres com idades entre 20 a 64 anos não procuraram os serviços de saúde nos últimos doze meses. Em relação às consultas médicas $3,9 \%$ das mulheres relataram que realizaram pelo menos uma nos últimos doze meses em contrapartida apenas 1,8\% dos homens ${ }^{9}$. O perfil dos homens que procuram tais serviços são indivíduos acompanhados por parceiras, e aqueles que possuem maior grau de instrução, 90.

Em 2008, o Ministério da Saúde criou a Política Nacional de Atenção Integral à Saúde do Homem (PNAISH), na tentativa de englobar esta população em consonância dos princípios do Sistema Único de Saúde (SUS), garantindo acesso e cuidado integral, além de prevenir agravos e promover saúde. Esta política possui em suas diretrizes a necessidade do conhecimento das doenças prevalentes nessa população, fortalecendo assim a rede de cuidados em saúde ${ }^{11,12}$.

Percebendo o crescimento do número de casos de neoplasias em todo o mundo, em específico o câncer de mama, este estudo se propõe a analisar as taxas de mortalidade de homens com câncer de mama em cada região do Brasil, com ênfase nos estados da Região Nordeste. 


\section{MÉTODO}

Trata-se de um estudo ecológico, temporal com abordagem quantitativa, onde foram coletados os número de óbitos da população masculina residente no Brasil por câncer de mama registrados entre os anos de 2001 a 2015, com ênfase na região do Nordeste, que compreende os estados de Alagoas, Bahia, Ceará, Maranhão, Paraíba, Pernambuco, Piauí, Rio Grande do Norte e Sergipe.

Foi utilizada a taxa de mortalidade por neoplasia mamária, classificado sob o código C50 da Classificação Estatística Internacional de Doenças e Problemas Relacionados com a Saúde (CID 10), segundo a localização primária do tumor.

Os dados foram coletados no mês de setembro de 2018 , através do site do atlas online de mortalidade do Instituto Nacional do Câncer (INCA) ${ }^{13}$ que usa os dados do Ministério da Saúde (MS) por meio do Sistema de Informação sobre Mortalidade (SIM) do sistema de informação DATASUS.

O presente estudo utilizou uma fonte de dados de domínio público, sendo dispensado do parecer do Comitê Ética de Pesquisa (CEP) obedecendo às normas éticas exigidas pela Resolução nº 466/2012 Conselho Nacional de Saúde (CNS).

\section{RESULTADO}

No Brasil, 1839 homens foram a óbito em razão do câncer de mama. A tabela 1 mostra como essa taxa de mortalidade se comportou nas 5 regiões geográficas do país. Observa-se que a mortalidade no Sudeste do Brasil ultrapassou as demais regiões com 49,7\% do número de óbitos entre os anos de 2001 a 2015 . Em contrapartida 0 Centro-Oeste demonstrou a menor taxa nacional, apresentando 4,6\% dos óbitos.

Tabela 1: Taxas de mortalidade por câncer de mama nas Regiões do Brasil e Brasil entre 2001 e 2015.

\begin{tabular}{lccc}
\hline REGIÃO & $\mathbf{N}^{\circ}$ MORTES & TAXA BRUTA & TAXA BRASIL \\
\hline CENTRO-OESTE & 115 & 0,11 & 0,15 \\
NORDESTE & 456 & 0,12 & 0,15 \\
NORTE & 85 & 0,07 & 0,12 \\
SUDESTE & 915 & 0,16 & 0,18 \\
SUL & 268 & 0,13 & 0,15 \\
BRASIL & 1839 & 0,13 & 0,16 \\
\hline
\end{tabular}

Fonte: Ministério da Saúde/ Sistema de Informação de Mortalidade - SIM. Taxas brutas e ajustadas pela população brasileira de 2010 , por 100.000 homens. 
Com relação à faixa etária dos indivíduos a idade mínima para o aparecimento de óbitos se deu entre 10 a 14 anos, entretanto os dados evidenciam que a mortalidade tende a aumentar exponencialmente a partir dos 30 a 39 anos de idade, chegando a 450 óbitos em homens entre 60 a 69 anos, para em seguida decair levemente na população masculina a partir dos 70 anos (tabela 2).

Tabela 2: Taxas de mortalidade por câncer de mama no Brasil entre 2001 e 2015, segundo faixas etárias.

\begin{tabular}{lcc}
\hline FAIXA ETÁRIA & ÓBITOS HOMENS & TAXA ESPECÍFICA HOMENS \\
\hline 00 a 04 & 0 & 0,00 \\
05 a 09 & 0 & 0,00 \\
10 a 14 & 1 & 0,00 \\
15 a 19 & 0 & 0,00 \\
20 a 29 & 12 & 0,00 \\
30 a 39 & 58 & 0,03 \\
40 a 49 & 180 & 0,11 \\
50 a 59 & 358 & 0,31 \\
60 a 69 & 450 & 0,64 \\
70 a 79 & 431 & 1,17 \\
80 ou mais & 349 & 2,38 \\
Idade ignorada & 0 & 0,00 \\
Total & 1839 & - \\
\hline
\end{tabular}

Fonte: Ministério da Saúde/ Sistema de Informação de Mortalidade - SIM. Taxas brutas e ajustadas pela população brasileira de 2010 , por 100.000 homens.

Quanto a região Nordeste, os estados do Ceará, Rio Grande do Norte e Sergipe se sobressaíram ao apontarem, respectivamente, as três maiores taxas de mortalidade da região (tabela 3). Na situação inversa, encontra-se o Maranhão, o qual se destacou por sua menor taxa, dos 456 óbitos ocorridos na região Nordeste, apenas 5,4\% ocorreram no estado. Tabela 3: Taxas de mortalidade por câncer de mama no Brasil no período de 2001 e 2015, entre os estados da região Nordeste.

\begin{tabular}{lccc}
\hline ESTADO & $\mathbf{N}^{\circ}$ MORTES & TAXA BRUTA & TAXA BRASIL \\
\hline ALAGOAS & 19 & 0,08 & 0,12 \\
BAHIA & 105 & 0,10 & 0,13 \\
CEARÁ & 102 & 0,17 & 0,21 \\
MARANHÃO & 25 & 0,05 & 0,08 \\
PARAÍBA & 36 & 0,13 & 0,15 \\
PERNAMBUCO & 83 & 0,13 & 0,16 \\
PIAUÍ & 20 & 0,09 & 0,11 \\
RIO GRANDE DO NORTE & 47 & 0,21 & 0,24 \\
SERGIPE & 19 & 0,13 & 0,18 \\
TOTAL & 456 & - & - \\
\hline
\end{tabular}

Fonte: Ministério da Saúde/ Sistema de Informação de Mortalidade - SIM. Taxas brutas e ajustadas pela população brasileira de 2010 , por 100.000 homens. 


\section{DISCUSSÃO}

Ao longo dos últimos anos houve um crescimento no número de casos de câncer de mama na população masculina. As maiores taxas de mortalidade encontram-se na América do Norte e Europa, com perspectivas de aumento devido à má qualidade de vida e a dificuldade no diagnóstico precoce ${ }^{14}$. O Brasil acompanha a tendência mundial quanto ao crescimento do número de óbitos por câncer de mama masculino ${ }^{15}$.

Algumas regiões do país apresentam taxas substancialmente maiores do que as demais como é o caso da região Sudeste, estudo semelhante encontrou resultados que comprovam esse achado. Enquanto no Sudeste o número de mortes por câncer de mama masculino chegou 749 mortes no Norte esse número caiu para 76 indivíduos ${ }^{16}$.

Alves ${ }^{17}$ ressalta que um dos principais motivos para esta disparidade entre regiões se dá por causa das dimensões continentais do país, de sua diversidade e também da desigualdade socioeconômica e política que coexistem em uma mesma cidade, fazendo-se necessário um movimento de descentralização da saúde para que as ações de promoção e prevenção contemplem todo o território

No Brasil, existe uma baixa cobertura populacional em relação ao câncer de mama e um extenso tempo para o diagnóstico. 0 perfil socioeconômico de pacientes com maior vulnerabilidade a desenvolver o câncer são os que possuem baixa renda, menores níveis escolares e não brancos, essas diferenças nas taxas de mortalidade encontradas podem ser relacionadas as limitações nos diagnósticos e tratamento existentes ${ }^{18}$.

Existem lacunas nas políticas de atenção voltadas à saúde do homem, as quais não conseguem atingir um quantitativo satisfatório do público alvo, principalmente nas regiões carentes onde ocorre dificuldade no acesso às informações e aos serviços de saúde. Ainda é preciso levar em consideração a subnotificação dos casos ou notificação tardia, o que pode gerar uma subestimação do número de ocorrências, dificultando as atividades de vigilância em saúde, avaliação e programação dos serviços para a real situação epidemiológica do país ${ }^{19}$.

Segundo o presente estudo, homens com idade entre 60 aos 69 anos foram os que, em maior número, evoluíram de forma fatal. A idade se mostra como um fator primordial desse aparecimento e a incidência maior é apresentada no intervalo de 70 anos $^{20}$. Resultados semelhantes foram encontrados no Paraguai, onde a média de maior incidência foi de 68 anos corroborando com o estudo apresentado ${ }^{21}$.

Seguindo a mesma tendência, Lattin et al. ${ }^{22}$ verificou que o diagnóstico da doença na população masculina ocorre, em média, entre 60 e 70 anos. Entretanto, um ensaio prospectivo de 20 anos em um hospital da Turkey verificou que a idade média dos homens acometidos foi de 59,8. Esse grande número diagnóstico em idosos demonstrado na literatura, principalmente quando feito de forma tardia, pode aumentar as chances de complicações acrescidos do câncer como metástases e morte ${ }^{23}$. 
Sabe-se que a cultura influencia na construção dos gêneros, e no Brasil, durante último século o cuidado foi atrelado ao feminino, esta visão pode influenciar negativamente o homem a procurar os serviços de saúde, comprometendo a este indivíduo o acesso ao tratamento precoce e diminuindo suas chances de cura, por isso a PNAISH deve identificar a heterogeneidade do gênero e incentivar sua participação em todas as fases de sua vida ${ }^{24}$.

A região Nordeste apresentou a terceira maior taxa de mortalidade proporcional por câncer de mama masculino. Dentro deste escopo, os estados do Ceará, Rio Grande do Norte e Sergipe apresentam as maiores taxas, que podem ser justificadas pelas diferenças regionais no acesso aos serviços de saúde e aspectos como pobreza, ausência de saneamento básico e menor nível de escolaridade. A falta de acesso à educação pode agir como barreira na procura dos homens e mulheres aos serviços de saúde, gerando consequências como diagnóstico tardio ou subnotificação ${ }^{25}$.

Os fatores de risco para o câncer de mama masculino são vastos e incluem não só os fatores externos/ambientais como consumo de hormônios, exposição a produtos tóxicos, mas também a mutações genéticas principalmente entre os genes BRCA1 e BRCA226,27. Uma doença de etiologia diversa que necessita de um olhar multifacetado para reconhecer não só o agravo em saúde, mas também como a construção ambiental/social do indivíduo pode influenciar positiva ou negativamente na busca por tratamento, principalmente quando observado que culturalmente o câncer de mama está atrelado ao feminino o que acarreta a esses homens implicações pessoais, psicológicas e sociais que dificultam a procura por serviços de saúde 28 .

Mesmo com as inúmeras ações da PNAISH para inserção do cuidado em saúde do homem nos serviços de saúde, ainda é escassa a procura desse público por motivos variados, como as longas jornadas de trabalho, medo de diagnósticos, vergonha e existência do estereótipo da masculinidade em ser invulnerável. O homem procura a equipe de Estratégia Saúde da Família (ESF) de forma pontual, para problemas de resolução imediata como recebimento de medicação, renovação de receitas e outras ações curativas centradas no modelo biomédico ${ }^{29}$.

\section{CONSIDERAÇÕES FINAIS}

O trabalho avaliou a taxa de mortalidade proporcional por câncer de mama masculino no período de 2001 a 2015 nas regiões geográficas do Brasil, com enfoque nos estados do Nordeste. Foi evidenciado que a taxa de mortalidade na região Sudeste se encontrou acima da média nacional. Quanto aos estados do Nordeste, destacaram-se o Ceará, Rio Grande do Norte e Sergipe como os que concentravam as maiores taxas de mortalidade da região e constatou-se que as chances de morte aumentam exponencialmente em indivíduos acima de 50 anos de idade. A limitação deste estudo foi a dificuldade em encontrar artigos sobre o tema, por se tratar de uma doença rara, poucos estudos investigaram a patologia e epidemiologia, tornando os dados epidemiológicos de difícil acesso, sobretudo quando se trata da população brasileira. 


\section{REFERÊNCIAS}

1. Korde LA, Zujewski JA, Kamin L, Giordano S, Domchek S, Anderson WF, et al. Multidisciplinary meeting on male breast cancer: summary and research recommendations. Journal of. Clinical Oncology. 2010; 28(12): $2114-22$.

2. Yousef AJA. Male Breast Cancer: Epidemiology and Risk Factors. Seminars in Oncology. 2017; 44(4), 267-72.

3. Ministério da Saúde. Instituto Nacional de Câncer, Causas e Prevenção - Estatísticas de Câncer. 2020. https://www.inca.gov.br/numeros-de-cancer. Acesso em 31 Mar, 2020.

4. Brinton LA, Cook MB, McCormack V, Johnson KC, Olsson H, Casagrande JT, et al. Anthropometric and hormonal risk factors for male breast cancer: male breast cancer pooling project results. JNCl: Journal of the National Cancer Institute. 2014; 106(3).

5. Ferlay J, Soerjomataram I, Dikshit R, Eser S, Mathers C, Rebelo M, et al. Cancer incidence and mortality worldwide: sources, methods and major patterns in GLOBOCAN 2012. International Journal of Cancer. 2015; 136(5): $359-86$.

6. Bray F, Jemal A, Grey N, Ferlay J, Forman D. Global cancer transitions according to the Human Development Index (2008-2030): a population-based study. The lancet oncology. 2012; 13(8):790-801.

7. Malta DC, Moura L, Prado RR, Escalante JC, Schmidt MI, Duncan BB. Mortalidade por doenças crônicas não transmissíveis no Brasil e suas regiões, 2000 a 2011. Epidemiologia e Serviços de Saúde. 2014; 23(4): 599-608.

8. Bidinotto DNPB, Simonetti JP, Bocchi SCM. A saúde do homem: doenças crônicas não transmissíveis e vulnerabilidade social. Revista Latino-Americana de Enfermagem. 2016; 24:1-8.

9. Brasil. Instituto Brasileiro de Geografia e Estatística. Pesquisa Nacional por Amostra de Domicílios: um panorama da saúde no Brasil. Acesso e utilização dos serviços, condições de saúde e fatores de risco e proteção à saúde 2008. 2010. 
10. Couto MT et al. O homem na atenção primária à saúde: discutindo (in)visibilidade a partir da perspectiva de gênero. Interface - Comunicação, Saúde, Educação. 2010;14(33):257-70.

11. Brasil. Ministério da Saúde. Secretaria de Atenção à Saúde. Departamento de Ações Programáticas Estratégicas. Política Nacional de Atenção Integral à Saúde do Homem: princípios e diretrizes. Brasília: Ministério da Saúde; 2008.

12. Cavalcanti JRD, Ferreira JA, Barros AH, Morais GSN, Trigueiro JVS, Torquato IMB. Assistência Integral a Saúde do Homem: necessidades, obstáculos e estratégias de enfrentamento. Escola Anna Nery. 2014; 18(4): 628-34.

13. Brasil. Instituto Nacional de Câncer. Atlas de Mortalidade. 2015 [acesso 15 jun 2018]; Disponível em: https://mortalidade.inca.gov.br/MortalidadeWeb/.

14. Hass P, Costa AP, Souza AP. Epidemiologia do câncer de mama em homens. Revista do Instituto Adolfo Lutz. 2009;68(3):476-81.

15. Barros HCS. Aspectos epidemiológicos da mortalidade por câncer de mama masculina no Brasil, no período de 1996 a 2013. [Dissertação] Maceió, Universidade Federal de Alagoas; 2016.

16. Maselli-Schoueri JH, Affonso-Kaufman FA, Sette CVM, Figueiredo FWS, Adami F. Time trend of breast cancer mortality in BRAZILIAN men: 10-year data analysis from 2005 to 2015. BMC Cancer. 2019;19(1):23.

17. Alves MO, Magalhães SCM, Coelho BA. A regionalização da saúde e a assistência aos usuários com câncer de mama. Saúde e Sociedade. 2017;26(1):141-54.

18. Vieira, RAC, Formenton A, Bertolini, SR. Breast cancer screening in Brazil. Barriers related to the health system. Revista da Associação Médica Brasileira. 2017;63(5):466-474.

19. Souza MA et al. Percepção dos profissionais de saúde sobre os fatores associados à subnotificação no Sistema Nacional de Agravos de Notificação (Sinan). Revista de Administração em Saúde. 2018;26:1-17. 
20. Ferzoco RM, Ruddy KJ. The Epidemiology of Male Breast Cancer. Current Oncology Reports. 2016;18(1):1-6.

21. Camejo N, Castillo C, Shiavone A, Alfonso AL, Amarillo D, Xavier F, et al. Características del cáncer de mama masculino en una población. Anales de la Facultad de Medicina, Universidad de la República, Uruguay. 2018;5(1):26-37.

22. Lattin Jr GE, Jesinger RA, Mattu R, Glassman LM. From the radiologic pathology archives: diseases of the male breast: radiologic-pathologic correlation. Radiographics. 2013;33(2):461-89.

23. Serarslan A, Gursel B, Okumus NO, Meydan D, Sullu Y, Gonullu G. Male Breast Cancer: 20 Years Experience of a Tertiary Hospital from the Middle Black Sea Region of Turkey. Asian Pacific Journal Of Cancer Prevention. 2015;16(5):6673-79.

24. Pereira J, Klein C, Meyer D E. PNAISH: uma análise de sua dimensão educativa na perspectiva de gênero. Saúde e Sociedade. 2019; 28(2): 132-146.

25. Nogueira SP, Mendonça JV, Pasqualette HAP. Câncer de mama em homens. Revista Brasileira de Mastologia. 2014;24(4):109-104.

26. Fentiman IS, Fourquet A, Hortobagyi GN. Male breast cancer. The Lancet. 2006; 367(9510):595-604.

27. Gennari R, Curigliano G, Fossa BAJ, Zurrida S, Renne G, Intra M, et al. Male breast cancer: A special therapeutic problem. International Journal of Oncology. 2004; 24 (3):663-70.

28. Amaral DED, Muniz RM, Cardoso DH, Noguez PT, Fagundes RF, Viegas AC. Câncer de mama masculino: 0 contexto do sobrevivente. Revista de enfermagem da UFPE on line. 2017; 11(5):1783-1790.

29. Lemos AP, Ribeiro C, Fernandes J, Bernardes K, Fernandes R. Saúde do homem: os motivos da procura dos homens pelos serviços de saúde. Revista de enfermagem da UFPE on line. 2017;11(supl 11):4546-53. 